\section{¿Son efectivas las intervenciones psicológicas para mejorar el control de la Diabetes Mellitus tipo 2 en adultos?: una revisión sistemática y metaanálisis}

\author{
ALEXIS GONZÁLEZ-BURBOA ${ }^{1,3,4, a, d,}$, CARLOS ACEVEDO COSSIO ${ }^{5, b, c,}$, \\ ALDO VERA-CALZARETTA ${ }^{1,3,4, \mathrm{~b}, \mathrm{e}}$, PATRICIA VILLASECA-SILVA ${ }^{1,3}$, \\ HANS MÜLLER-ORTIZ ${ }^{2,4}$, DARÍO PÁEZ ROVIRA ${ }^{6,8, b, e}$, \\ CRISTIAN PEDREROS ROSALES ${ }^{2,4}$, ÁNGEL ALBERQUILLA \\ MENÉNDEZ-ASENJO ${ }^{7}$, ÁNGEL OTERO PUIME ${ }^{7}$
}

\section{Psychological interventions for patients with type 2 diabetes mellitus. A systematic review and meta-analysis}

Background: Affordable interventions to improve metabolic control of Type 2-Diabetes Mellitus are increasingly necessary. Aim: To review systematically the existing literature on the effects of psychological interventions on Type-2 Diabetes Mellitus compensation. Material and Methods: We performed a systematic literature review and meta-analysis on the effectiveness of psychological interventions implemented for Type-2 Diabetes Mellitus patients. Research included the following electronic databases: PubMed, Bireme, Web of Science, SciELO, Embase, EBSCOhost, SCOPUS, Psychology Database. Results: Most studies showed a decrease in the level of glycated hemoglobin after interventions, which applied different initiatives complementary to standard medical treatment. Mainly, these interventions encompassed training for self-monitoring and control of diabetes based on cognitive behavioral psychology, counseling, self-assessment and physical-spiritual work based on transpersonal psychology. Conclusions: Psychological tools could be an adjunct to the standard medical treatment for patients with Type-2 Diabetes Mellitus, reducing glycated hemoglobin levels and improving self-regulation, disease awareness and adherence from the self-efficacy perception perspective.

(Rev Med Chile 2019; 147: 1423-1436)

Key words: Diabetes Mellitus, Type 2; Glycated Hemoglobin A; Psychology.
${ }^{1}$ Departamento de Salud Pública, Facultad de Medicina, Universidad de Concepción. Concepción, Chile.

${ }^{2}$ Departamento de Medicina Interna, Facultad de Medicina, Universidad de Concepción. Concepción, Chile.

${ }^{3}$ Programa de Salud y Medicina Familiar, Facultad de Medicina, Universidad de Concepción. Concepción, Chile.

${ }^{4}$ Unidad Docente Asistencial, Hospital Las Higueras, Facultad de Medicina,

Universidad de Concepción. Concepción, Chile.

${ }^{5}$ Departamento de Educación, Universidad Tecnológica de Chile INACAP, Chile. ${ }^{6}$ Departamento de Psicología Social y Metodología, Facultad de Psicología, Universidad del País Vasco, España. ${ }^{7}$ Departamento de Medicina Preventiva y Salud Pública, Facultad de Medicina, Universidad Autónoma de Madrid, España. ${ }^{8}$ Facultad de Educación y Ciencias

Sociales, Universidad Andrés Bello. Chile.

aMatrón.

bPsicólogo.

"MSc.

${ }^{d}$ Candidato a Doctor en Epidemiología y Salud Pública por la Universidad Autónoma de Madrid, España. ePhD.

Financiamiento: Este estudio se enmarca dentro del proyecto VRID 217.089.0061.0IN financiado por la Vicerrectoría de Investigación y Desarrollo de la Universidad de Concepción, Chile. No existen conflictos de interés con la fuente de financiamiento de esta investigación.

Recibido el 7 de febrero de 2019, aceptado el 15 de noviembre de 2019.

Correspondencia a: Aldo Vera-Calzaretta

Departamento de Salud Pública. Facultad de Medicina. Universidad de Concepción. Avda. Chacabuco esquina Janequeo $s / n$, $6^{\circ}$ piso. Concepción, Chile. avera.calzaretta@gmail.com
L a diabetes representa una de las mayores emergencias de salud del siglo XXI, estimándose que al año 2040 10,4\% de la población mundial de 20 a 79 años tendrá esta enfermedad ${ }^{1}$. El problema es particularmente grave en América
Central y del Sur donde habría casi 28 millones de personas con diabetes, proyectándose más de 48 millones para el 2045, situación que constituye uno de los mayores incrementos de esta enfermedad en el mundo ${ }^{2}$. 
Chile, en lo que respecta al grupo de personas entre 20 a 79 años, se sitúa en el segundo lugar de Latinoamérica ${ }^{1}$. Según datos disponibles ${ }^{3}$ la prevalencia de diabetes en la población chilena es de $9,4 \%$ en población mayor a 15 años, de los cuales, $52,1 \%$ recibe algún tratamiento farmacológico o no farmacológico.

Dado este escenario, en las últimas décadas el cuidado de la cronicidad ha evolucionado desde enfoques que consideraban solo la biología hacia enfoques que han ido incorporando nuevas formas de abordar el manejo de la enfermedad con una perspectiva más integral ${ }^{4,5}$. En este sentido, se ha dado especial atención al desarrollo de modelos y estrategias orientadas al fortalecimiento de la autogestión de las personas, al apoyo a la toma de decisiones y a la mejora de aspectos relacionados con la organización de los sistemas sanitarios, entre otras ${ }^{6-8}$. Esto ha permitido una apertura hacia la incorporación de intervenciones complementarias al tratamiento médico que permiten apoyar y acompañar a las personas que padecen una enfermedad crónica ${ }^{9-11}$.

Así, en el último tiempo ha cobrado relevancia el estudio de la efectividad de intervenciones implementadas en personas con diabetes, cuya forma más frecuente es la Diabetes Mellitus tipo $2-\mathrm{DM} 2{ }^{-1}$.

La literatura que reporta intervenciones aplicadas a personas con DM2, posee una abundante producción de estudios cuyo propósito ha sido establecer alguna relación entre variables psicológicas y el nivel de hemoglobina glicada -HbAlc-, parámetro que es ampliamente utilizado para referirse al nivel de compensación de la enfermedad ${ }^{11-14}$. En esta línea, el tipo de abordaje psicológico utilizado para intervenir estos pacientes se ha estudiado en algunos trabajos $^{13,14}$, por ejemplo, para determinar el efecto a corto, mediano y largo plazo de la psicología cognitivo conductual sobre el nivel de $\mathrm{HbAlc}^{12}$. No obstante, es relevante seguir generando evidencia respecto de intervenciones de bajo costo que puedan ser ampliamente extendidas en el escenario de cuidados de las personas que tienen esta enfermedad.

El propósito de este estudio fue revisar sistemáticamente y sintetizar la literatura existente acerca del efecto que las intervenciones psicológicas realizadas en pacientes con DM2 tendrían sobre el control de la enfermedad.

\section{Método}

\section{Diseño}

Se llevó a cabo una revisión sistemática y metaanálisis de estudios que reportaron la efectividad de intervenciones enfocadas a pacientes con DM2, que incorporaron la implementación de una intervención psicológica como parte de su diseño. Para la realización de este trabajo, se siguieron los criterios establecidos por la guía PRISMA (Preferred Reporting Items for Systematic Reviews and Meta-Analyses) $)^{15}$.

\section{Proceso de búsqueda de la literatura}

La búsqueda de los estudios fue realizada en las siguientes bases de datos electrónicas: PubMed, Bireme, Web of Science, SciELO, Embase, EBSCOhost, SCOPUS, Psychology Database, desde sus inicios hasta el 31 de diciembre de 2018. Se utilizaron los operadores boléanos AND y OR, y para las bases en inglés, los términos $\mathrm{MeSH}$ (Medical Subjects Headings) "Diabetes Melltius, Type 2" y "Glycated Hemoglobin A". Además, se utilizaron los términos libres "psychological interventions", "psychological intervention", "glycated hemoglobin" y "diabetes mellitus type 2 ". Específicamente, en la base Embase se aplicaron los límites de búsqueda "full text", "human", "english language", "article" y "adult $<18$ to 64 years $>$ or aged $<65+$ years $>$ ". Además, se especificó en la estrategia de búsqueda: NOT (("Diabetes Mellitus, Type 1" [Mesh]) OR diabetes mellitus type 1)), sin términos relacionados. Para el caso de Psychology Database se aplicaron los filtros "Texto completo", "Adulto (de 19 a 44 años)", "Persona de mediana edad (de 45 a 64 años)", "Tercera edad (65+ años)", "Tercera edad (80+ años)”, "Revistas científicas", "Artículo".

Para el caso de las bases de datos en español, se utilizaron los términos DeCS (Descriptores en Ciencias de la Salud) "Diabetes Mellitus Tipo 2", "intervención* psicológica ${ }^{\star}$ " "hemoglobina glicada". A continuación se presenta el flowchart del proceso de búsqueda bibliográfica (Figura 1).

\section{Elegibilidad de los estudios y extracción de los datos}

Se incluyeron estudios experimentales, que evaluaron el efecto de intervenciones psicológicas, implementadas en pacientes con DM2, sobre los niveles de HbA1c. Como criterios de elegibilidad de los estudios, se consideró que estos reportasen: 


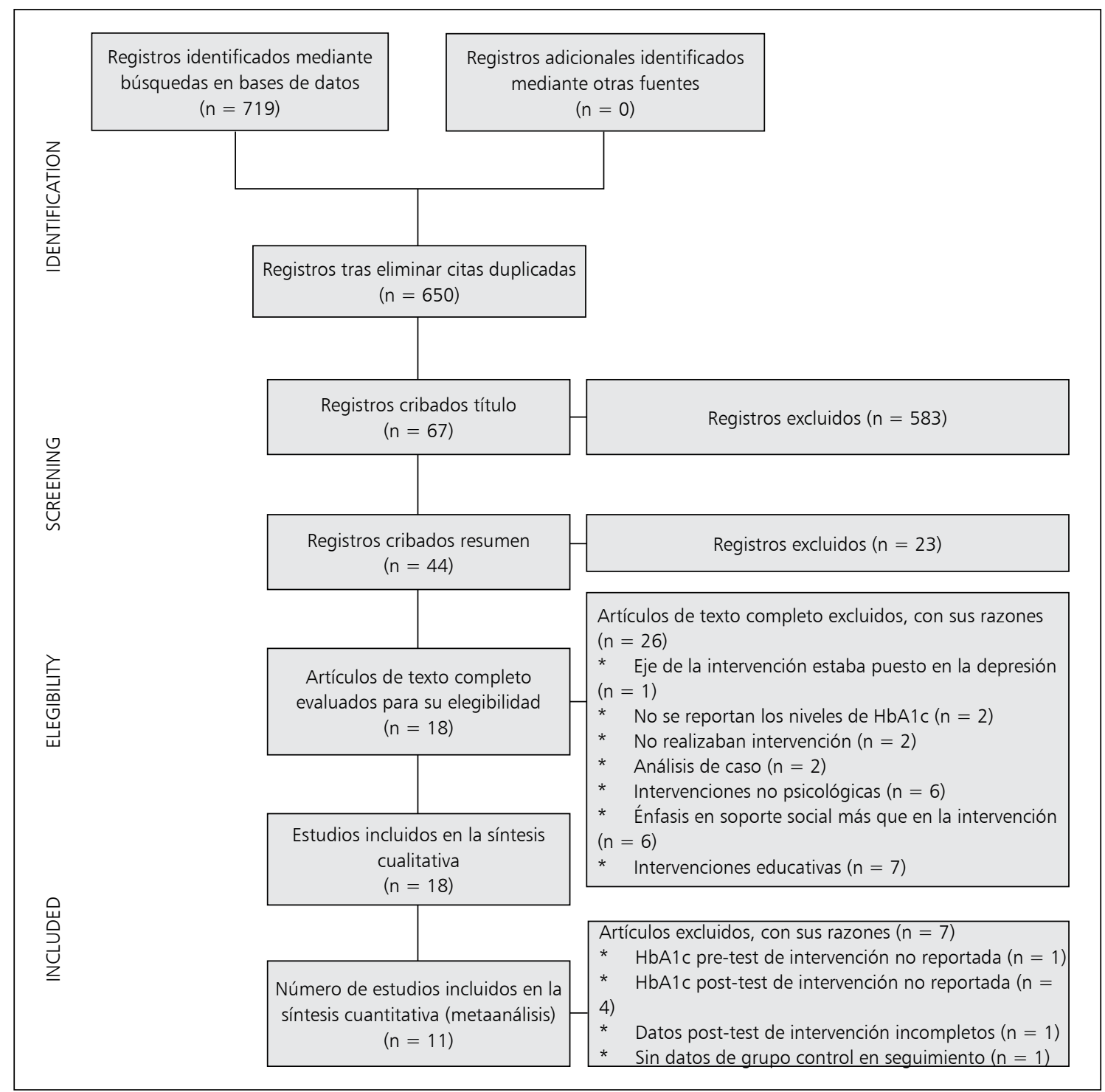

Figura 1. Flow chart PRISMA de los estudios seleccionados.

sujetos con una edad igual o mayor a 18 años; con diabetes mellitus tipo 2; la implementación de intervenciones psicológicas; $y$ tener como una de sus variables de resultado el nivel de HbAlc.

Respecto a las intervenciones implementadas, los modelos de abordaje psicológico fueron clasificados en las siguientes categorías: consejería ${ }^{16,17}$, psicología cognitivo conductual ${ }^{18-21}$ y psicología transpersonal ${ }^{22-25}$.

Los datos fueron extraídos por dos revisores independientes.

\section{Evaluación de la calidad metodológica}

Los trabajos incluidos en la síntesis cualitativa de este estudio, fueron revisados por dos evaluadores independientes. Para este efecto, se utilizó la escala de Jadad ${ }^{26}$ que valora los siguientes criterios en los ensayos clínicos: aleatorización, enmascaramiento y perdidas de seguimiento. Esta escala consta de 3 ítems cuya respuesta correcta equivale a 1 punto, e incorrecta a 0 puntos. Además, consta de dos preguntas adicionales que indagan la idoneidad de los procesos de aleatorización y 
enmascaramiento. La puntuación total de esta escala va de 0 a 5 puntos, estableciéndose que la obtención de un puntaje más alto refleja una mejor calidad metodológica del estudio.

El nivel de acuerdo entre los evaluadores fue establecido mediante la prueba de concordancia de Kappa ${ }^{27}$. Además, para resolver aquellos casos en los cuales no hubo acuerdo, se incluyó la opinión del autor principal como tercer evaluador independiente.

Para evaluar el riesgo de sesgo de publicación se utilizó la representación gráfica funnel plot. Además, se realizó un análisis de regresión de Egger y se calculó la N de seguridad de Rosenthal ${ }^{28}$.

\section{Análisis estadístico}

Para la realización del metaanálisis, se extrajeron tanto de los grupos de intervención y control, los siguientes datos: media, desviación estándar y tamaño de los grupos.

En la síntesis de los datos, se consideró adecuado un índice de heterogeneidad $-\mathrm{I}^{2}-$ entre $0 \%$ y $40 \%$, moderado entre $30 \%$ y $60 \%$, sustancial entre 50 y $90 \%$, y considerable entre $75 \%$ y $100 \%{ }^{29}$.

En el análisis, se calculó la diferencia de medias estandarizada. Dada la heterogeneidad considerable del metaanálisis global (Figura 2) se realizó un análisis de subgrupos según tipo de intervención psicológica y origen de los estudios, mediante un modelo de efectos aleatorios ${ }^{30}$. Además, se realizó una meta-regresión utilizando como variable de ajuste la duración de la intervención.
Los datos obtenidos fueron analizados con el software Review Manager V.5.3. y el Software Comprehensive Meta-Analysis (CMA). La significancia estadística para este estudio se estableció con un valor $\mathrm{p} \leq 0,05$.

\section{Resultados}

\section{Características de los estudios}

Se identificaron 18 trabajos, de los cuales, 6 fueron realizados en Estados Unidos de Norteamérica $^{31-36}, 3$ en Irán ${ }^{37-39}, 2$ en Países Bajos ${ }^{36,37,40,41}$. Además, se encontraron trabajos provenientes de China ${ }^{42}$, Canadá ${ }^{43}$, Alemania ${ }^{44}$, Reino Unido $^{45}$, Francia ${ }^{46}$, Taiwán ${ }^{47}$ y Grecia ${ }^{48}$. Del total de investigaciones realizadas, 10 utilizaron alguna intervención derivada de la psicología cognitivo conductual $^{32-34,36-38,40,43,45,48}$, consistente, principalmente, en entrenamientos para el auto-monitoreo y control de la diabetes. Estos estudios, además, involucraron al personal de salud en la atención integral de los pacientes. La segunda línea de intervención psicológica más frecuente, reportada en 6 estudios, fue la de las consejerías ${ }^{35,39,41,42,44,47}$, principalmente enfocadas a la inclusión de herramientas de comunicación que permitieron un contacto más fluido y dirigido entre la institución y el paciente. En específico, fueron intervenciones en las que se hizo un acompañamiento paralelo al tratamiento de la enfermedad que abarcaron, desde las concepciones sobre ésta, hasta la motivación

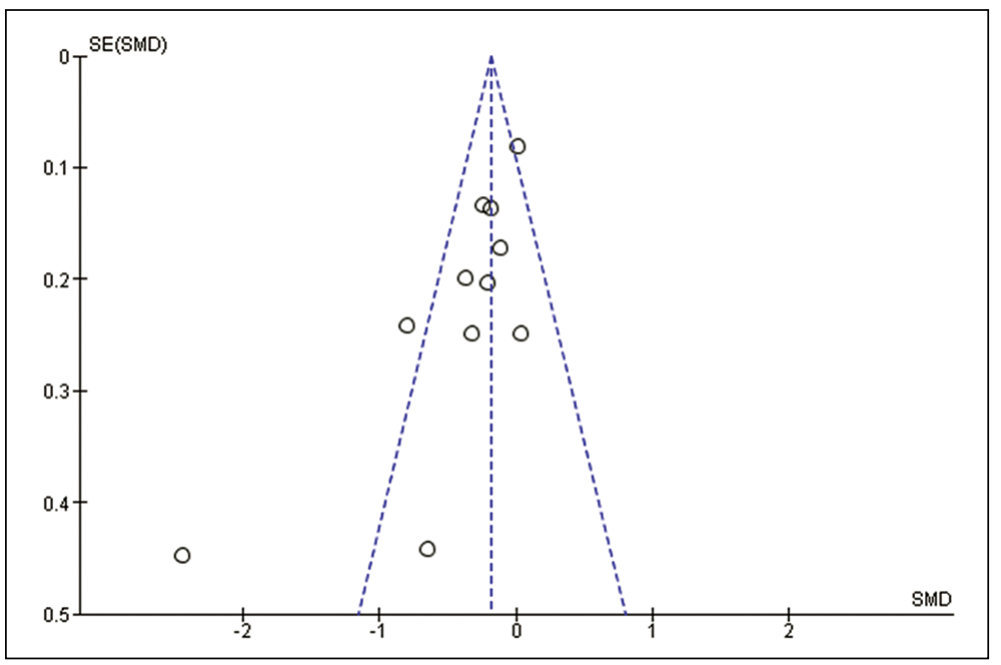

Figura 2. Funnel plot de los estudios incluidos en el metaanálisis. 
para enfrentar el tratamiento. Finalmente, aquellas intervenciones que se enfocaron en aspectos relativos a la autovaloración, trabajo afectivo directo o trabajo físico-espiritual basado en filosofías orientales, se sistematizaron bajo la mirada de la psicología transpersonal $^{31,46}$.

Las características de cada estudio se presentan a continuación en la Tabla 1.

\section{Evaluación de la calidad metodológica y sesgo de publicación}

Las evaluaciones independientes de la calidad metodológica de los artículos, lograron un nivel de acuerdo moderado $(\kappa=0,471 ; p<0,001)$. En aquellos casos en los cuales hubo desacuerdo, se buscó consenso mediante la inclusión de la evaluación del investigador principal que operó como tercer evaluador independiente. De esta forma, diez artículos fueron catalogados con un alto nivel de calidad ${ }^{32,33,35,37-40,42,43,47}$ y ocho con baja calidad $^{31,34,36,41,44,46-48}$ ) (Tabla 1).

$\mathrm{Al}$ evaluar el sesgo de publicación, el funnel plot y el análisis de regresión ( $\mathrm{p}=0,02)$, sugirieron un riesgo de existencia de este. No obstante lo anterior, el número mínimo de estudios para anular el efecto de la intervención fue alto $(\mathrm{N}$ de Rosenthal $=75$ ).

\section{Síntesis cuantitativa}

De los 18 estudios descritos previamente, los resultados de 11 trabajos fueron considerados en el metaanálisis ${ }^{32,33,35-38,40,42-44,47}$. Los estudios descartados, no reportaban los valores de HbAlc pre-test ${ }^{39}$ y post-test ${ }^{31,45,46,48}$ en el grupo de inter- vención, tenían datos post-test incompletos ${ }^{41}$, o no contaban con datos de seguimiento del grupo control $^{34}$.

El metaanálisis global mostró una reducción de los niveles de $\mathrm{HbAlc}$ en quienes recibieron una intervención psicológica (Dif. $=-0,35 ;-0,57$, $-0,12$ ). A pesar de esto, se observó un nivel de heterogeneidad considerable de los estudios $\left(\mathrm{I}^{2}=76 \%\right.$; $\mathrm{p}<0,001$ ) (Figura 3).

Dado lo anterior, se realizó un primer análisis de subgrupo considerando como variable de clasificación el tipo de intervención psicológica recibida. En el grupo de estudios que reportaron una intervención basada en la psicología cognitivo conductual ${ }^{32,33,36-38,40,43}$, si bien se apreció una reducción de los niveles de $\mathrm{HbAlc}$ (Dif. $=-0,47$; $-0,86,-0,07)$, estos presentaron una considerable heterogeneidad $\left(\mathrm{I}^{2}=78 \% ; \mathrm{p}<0,001\right)$. En cambio, en el grupo de estudios que reportaron la implementación de consejerías ${ }^{35,42,44,47}$, no se observó un cambio significativo $(\mathrm{p}=0,08)$. No fueron incluidos en este análisis los estudios que implementaron intervenciones basadas en psicología transpersonal debido a que presentaron datos faltantes ${ }^{31,46}$ (Figura 4).

En el segundo análisis de subgrupo, al considerar el origen de los estudios, solo en aquellos provenientes de América del Norte ${ }^{32,33,35,36,43}$, la disminución del nivel de $\mathrm{HbAlc}$ en las personas intervenidas fue estadísticamente significativa (Dif. $=-0,35 ;-0,61,-0,09)$. Los estudios provenientes de Asia ${ }^{42,47}$, Países Árabes ${ }^{37,38}$ y Europa ${ }^{40,44}$, no mostraron resultados significativos $(\mathrm{p}=0,58$; $\mathrm{p}=0,32 ; \mathrm{p}=0,24$, respectivamente).

\begin{tabular}{|c|c|c|c|c|c|c|c|c|c|c|}
\hline \multirow[b]{2}{*}{ Study or Subgroup } & \multicolumn{3}{|c|}{ Intervention } & \multicolumn{3}{|c|}{ Control } & \multicolumn{2}{|c|}{ Std. Mean Difference } & \multirow{2}{*}{\multicolumn{2}{|c|}{$\begin{array}{l}\text { Std. Mean Difference } \\
\text { IV, Random, } 95 \% \mathrm{Cl}\end{array}$}} \\
\hline & Mean & SD & Total & Mean & SD & Total & Weight & IV, Random, $95 \% \mathrm{Cl}$ & & \\
\hline Aikens, 1997 & 10.2 & 1.6 & 12 & 11.3 & 1.7 & 10 & $4.6 \%$ & $-0.64[-1.51,0.22]$ & & - \\
\hline Browning, 2016 & 6.67 & 2.09 & 312 & 6.64 & 1.59 & 296 & $12.8 \%$ & $0.02[-0.14,0.18]$ & & \\
\hline Chen, 2012 & 8.16 & 1.73 & 104 & 8.48 & 1.78 & 110 & $11.5 \%$ & $-0.18[-0.45,0.09]$ & & \\
\hline Ghezeljeh, 2017 & 7.39 & 1.35 & 30 & 7.35 & 1.35 & 35 & $8.4 \%$ & $0.03[-0.46,0.52]$ & & \\
\hline Gregg, 2007 & 7.47 & 1.46 & 36 & 8.07 & 2.22 & 30 & $8.4 \%$ & $-0.32[-0.81,0.17]$ & & \\
\hline Grey, 2000 & 7.5 & 1.1 & 41 & 8.5 & 1.4 & 34 & $8.6 \%$ & $-0.80[-1.27,-0.32]$ & & \\
\hline Lamers, 2011 & 7.3 & 0.2 & 20 & 7.8 & 0.2 & 17 & $4.5 \%$ & $-2.45[-3.32,-1.57]$ & & \\
\hline Shayeghian, 2016 & 7.03 & 1.52 & 53 & 7.61 & 1.56 & 50 & $9.7 \%$ & $-0.37[-0.76,0.02]$ & & \\
\hline Siebolds, 2005 & 7.47 & 1.27 & 113 & 7.81 & 1.52 & 110 & $11.5 \%$ & $-0.24[-0.51,0.02]$ & & \\
\hline Wayne, 2015 & 7.88 & 1.17 & 48 & 8.13 & 1.27 & 49 & $9.6 \%$ & $-0.20[-0.60,0.20]$ & & \\
\hline Weinger, 2011 & 8.45 & 1.3 & 66 & 8.6 & 1.3 & 70 & $10.5 \%$ & $-0.11[-0.45,0.22]$ & & \\
\hline Total $(95 \% \mathrm{Cl})$ & & & 835 & & & 811 & $100.0 \%$ & $-0.35[-0.57,-0.12]$ & & \\
\hline \multicolumn{9}{|c|}{$\begin{array}{l}\text { Heterogeneity. } \text { Tau }^{2}=0.10 ; \mathrm{Chi}^{2}=41.55, \mathrm{df}=10(P<0.00001) ; I^{2}=76 \% \\
\text { Test for overall effect: } Z=3.00(P=0.003)\end{array}$} & $\begin{array}{ccc}-2 & -1 & 1 \\
\text { Favours [Intervention] }\end{array}$ & $\begin{array}{cc}1 & 1 \\
\text { Favours [Control] }\end{array}$ \\
\hline
\end{tabular}

Figura 3. Metaanálisis global del efecto de las intervenciones psicológicas sobre la reducción de la HbA1c. 
Tabla 1. Características de los estudios incluidos en la revisión sistemática

\begin{tabular}{|c|c|c|c|c|c|}
\hline $\begin{array}{l}\text { Año y } \\
\text { referencia }\end{array}$ & País & $\begin{array}{c}\text { n de } \\
\text { participantes, } \\
\% \text { hombres }\end{array}$ & $\begin{array}{c}\text { Media de } \\
\text { edad } \\
\text { (DE o rango), } \\
\text { años }\end{array}$ & $\begin{array}{c}\text { Modelo (tipo) y } \\
\text { duración de la terapia } \\
\text { en el grupo de } \\
\text { intervención }\end{array}$ & $\begin{array}{l}\text { Régimen (sesiones) en el } \\
\text { grupo de intervención y } \\
\text { especialidad del terapista }\end{array}$ \\
\hline $\begin{array}{l}\text { Ghezeljeh, } \\
2017^{38}\end{array}$ & Irán & 65 (NR) & $54,31 \pm 5,85$ & $\begin{array}{l}\text { Capacitación durante dos } \\
\text { días en una semana de } 40 \text { - } \\
60 \text { min sobre la técnica de } \\
\text { relajación. Seguimiento por } \\
12 \text { semanas }\end{array}$ & $\begin{array}{l}2 \text { Sesiones diarias de } 15-20 \text { min } \\
\text { de ejercitación autodirigido, en } \\
\text { un proceso de } 12 \text { semanas con } \\
\text { monitoreo telefónico, instructor } \\
\text { en Jacob's PMR training es uno } \\
\text { de los investigadores }\end{array}$ \\
\hline $\begin{array}{l}\text { Browning, } \\
2016^{42}\end{array}$ & China & $711(47,3 \%)$ & $63,80 \pm 8,30$ & $\begin{array}{l}\text { Coach telefónico y per- } \\
\text { sonal, sumado a terapia } \\
\text { estándar por } 12 \text { meses }\end{array}$ & $\begin{array}{l}2 \text { llamadas y dos visitas men- } \\
\text { suales por los primeros } 3 \text { meses; } \\
\text { una llamada y dos visitas men- } \\
\text { suales en el segundo trimestre; } \\
\text { una llamada y una visita men- } \\
\text { sual por un semestre, personal } \\
\text { clínico capacitado (psicólogo, } \\
\text { enfermera, médico) }\end{array}$ \\
\hline $\begin{array}{l}\text { Ebrahimi, } \\
2016^{39}\end{array}$ & Irán & $103(41,4 \%)$ & $48,15 \pm 6,52$ & $\begin{array}{l}5 \text { a } 6 \text { reuniones de entre } \\
60-90 \text { min semanales por } \\
8 \text { semanas }\end{array}$ & $\begin{array}{l}\text { Psicoeducación para el } \\
\text { empowerment, dado por } \\
\text { enfermeras y endocrinólogos } \\
\text { capacitados }\end{array}$ \\
\hline $\begin{array}{l}\text { Shayeghian, } \\
2016^{37}\end{array}$ & Irán & $100(40 \%)$ & $55,44 \pm 8,44$ & $\begin{array}{l}\text { Programa de intervención } \\
\mathrm{ACT} \text { en } 10 \text { sesiones }\end{array}$ & $\begin{array}{l}\text { Entrenamiento psicológico } \\
\text { en } 10 \text { sesiones orientado a la } \\
\text { aceptación y el compromiso con } \\
\text { el tratamiento, complementario } \\
\text { a educación sobre diabetes }\end{array}$ \\
\hline $\begin{array}{l}\text { Wayne, } \\
2015^{43}\end{array}$ & Canadá & $97(28 \%)$ & $53,20 \pm 11,30$ & $\begin{array}{l}\text { Entrenamiento conductual } \\
\text { por } 6 \text { meses }\end{array}$ & $\begin{array}{l}\text { Se estableció individualmente } \\
\text { un set de metas en relación a } \\
\text { necesidades detectadas por dos } \\
\text { autores, orientadas y acompa- } \\
\text { ñadas por ellos } 24 / 7 \text { mediante } \\
\text { contacto telefónico }\end{array}$ \\
\hline $\begin{array}{l}\text { Koloverou, } \\
2014(48)\end{array}$ & Grecia & $53(81.1 \%)$ & $61,83 \pm 7,40$ & $\begin{array}{l}\text { Entrenamiento en res- } \\
\text { piración de relajación y } \\
\text { relajamiento muscular } \\
\text { progresivo, tratamiento } \\
\text { por } 8 \text { semanas }\end{array}$ & $\begin{array}{l}\text { Sesiones auto dirigidas de } 10 \\
\text { min de respiración de relaja- } \\
\text { ción y } 15 \text { min de relajamiento } \\
\text { muscular progresivo durante } 8 \\
\text { semanas }\end{array}$ \\
\hline $\begin{array}{l}\text { Fall, } \\
2013^{46}\end{array}$ & Francia & $80(47 \%)$ & $62,10 \pm 11,70$ & $\begin{array}{l}\text { Una sesión de conversa- } \\
\text { ción y autorreporte }\end{array}$ & $\begin{array}{l}\text { Intervención transversal en dos } \\
\text { grupos control y amenaza }\end{array}$ \\
\hline $\begin{array}{l}\text { Putiri, } \\
2012^{32}\end{array}$ & $\begin{array}{l}\text { Estados } \\
\text { Unidos }\end{array}$ & $32(42 \%)$ & $56,30 \pm 8,10$ & $\begin{array}{l}\text { Meditación Yi Ren Qigong } \\
\text { grupal por } 12 \text { semanas }\end{array}$ & $1 \mathrm{~h}$ a la semana por 12 semanas \\
\hline $\begin{array}{l}\text { Chen, } \\
2012^{47}\end{array}$ & Taiwán & $215(49.8 \%)$ & $58,92 \pm 10,22$ & $\begin{array}{l}\text { Entrevista motivacional } \\
\text { única }\end{array}$ & $\begin{array}{l}\text { Entrevista motivacional de Mi- } \\
\text { ller and Rollnick de } 45-60 \text { min, } \\
\text { ejecutada por especialistas en } \\
\text { enfermería y tratamiento de } \\
\text { diabetes }\end{array}$ \\
\hline
\end{tabular}


Tabla 1. Características de los estudios incluidos en la revisión sistemática (continuación)

\begin{tabular}{|c|c|c|c|c|c|c|c|}
\hline $\begin{array}{l}\text { Año y } \\
\text { referencia }\end{array}$ & $\begin{array}{l}\text { Modelo (tipo) } \\
\text { y duración } \\
\text { de la terapia } \\
\text { en el grupo } \\
\text { control }\end{array}$ & $\begin{array}{c}\text { Régimen } \\
\text { (sesiones) } \\
\text { en el grupo } \\
\text { control y } \\
\text { especialidad } \\
\text { del terapista }\end{array}$ & $\begin{array}{c}\text { Segui- } \\
\text { miento, } \\
\text { meses }\end{array}$ & $\begin{array}{c}\text { Categoría } \\
\text { intervención }\end{array}$ & $\begin{array}{c}\text { Media (DE) \% } \\
\text { de hemoglo- } \\
\text { bina glicada } \\
\text { en la línea } \\
\text { base }\end{array}$ & $\begin{array}{c}\text { Media (DE) \% } \\
\text { de hemoglo- } \\
\text { bina glicada } \\
\text { en segui- } \\
\text { miento }\end{array}$ & $\begin{array}{c}\text { Calidad } \\
\text { metodo- } \\
\text { lógica }\end{array}$ \\
\hline $\begin{array}{l}\text { Ghezeljeh, } \\
2017^{38}\end{array}$ & $\begin{array}{c}\text { Terapia están- } \\
\text { dar }\end{array}$ & NR & 0 meses & $\begin{array}{l}\text { Psicología } \\
\text { cognitivo- } \\
\text { conductual }\end{array}$ & $8,41 \pm 1,65$ & $7,39 \pm 1,35$ & Alta \\
\hline $\begin{array}{l}\text { Browning, } \\
2016^{42}\end{array}$ & $\begin{array}{l}\text { Terapia están- } \\
\text { dar } 12 \text { meses }\end{array}$ & $\begin{array}{l}1 \text { sesión } \\
\text { mensual por } \\
12 \text { meses }\end{array}$ & 0 meses & Consejería & $10,60 \pm 2,09$ & $6,67 \pm 1,69$ & Alta \\
\hline $\begin{array}{l}\text { Ebrahimi, } \\
2016^{39}\end{array}$ & $\begin{array}{c}\text { Terapia están- } \\
\text { dar }\end{array}$ & NR & NR & Consejería & NR & $7,75 \pm 1,29$ & Alta \\
\hline $\begin{array}{l}\text { Shayeghian, } \\
2016^{37}\end{array}$ & $\begin{array}{c}\text { Educación so- } \\
\text { bre diabetes }\end{array}$ & 1 sesión de 2 h & 3 meses & $\begin{array}{l}\text { Psicología } \\
\text { cognitivo- } \\
\text { conductual }\end{array}$ & $7,46 \pm 1,66$ & $7,03 \pm 1,52$ & Alta \\
\hline $\begin{array}{l}\text { Wayne, } \\
2015^{43}\end{array}$ & $\begin{array}{l}\text { Coaching } \\
\text { en salud sin } \\
\text { acompaña- } \\
\text { miento por } \\
\text { smartphone }\end{array}$ & $\begin{array}{c}6 \text { meses } \\
\text { de llamadas } \\
\text { semanales }\end{array}$ & NR & $\begin{array}{l}\text { Psicología } \\
\text { cognitivo- } \\
\text { conductual }\end{array}$ & $8,69 \pm 1,32$ & $7,88 \pm 1,17$ & Alta \\
\hline $\begin{array}{l}\text { Koloverou, } \\
2014 \text { (48) }\end{array}$ & $\begin{array}{c}\text { Terapia están- } \\
\text { dar }\end{array}$ & NR & 0 meses & $\begin{array}{l}\text { Psicología } \\
\text { cognitivo- } \\
\text { conductual }\end{array}$ & $6,51 \pm 0,63$ & NR & Baja \\
\hline $\begin{array}{l}\text { Fall, } \\
2013^{46}\end{array}$ & $\begin{array}{l}\text { Una sesión de } \\
\text { conversación y } \\
\text { autorreporte }\end{array}$ & $\begin{array}{l}\text { Dos grupos con- } \\
\text { trol: Evocación } \\
\text { de emoción po- } \\
\text { sitiva y negativa }\end{array}$ & 0 meses & $\begin{array}{l}\text { Psicología } \\
\text { transpersonal }\end{array}$ & $8,04 \pm 1,35$ & NR & Baja \\
\hline $\begin{array}{l}\text { Putiri, } \\
2012^{32}\end{array}$ & $\begin{array}{c}\text { PRT, } 12 \text { sema- } \\
\text { nas }\end{array}$ & $\begin{array}{c}1 \text { vez a la } \\
\text { semana por } 12 \\
\text { semanas }\end{array}$ & 0 meses & $\begin{array}{l}\text { Psicología } \\
\text { transpersonal }\end{array}$ & $8,80 \pm 1,10$ & NR & Baja \\
\hline $\begin{array}{l}\text { Chen, } \\
2012^{47}\end{array}$ & $\begin{array}{c}\text { Educación so- } \\
\text { bre diabetes }\end{array}$ & $1 \mathrm{~h}$ semanal & 3 meses & Consejería & $8,92 \pm 2,17$ & $8,16 \pm 1,73$ & Alta \\
\hline
\end{tabular}


Tabla 1. Características de los estudios incluidos en la revisión sistemática (continuación)

\begin{tabular}{|c|c|c|c|c|c|}
\hline $\begin{array}{l}\text { Año y } \\
\text { referencia }\end{array}$ & País & $\begin{array}{c}\text { n de } \\
\text { participantes, } \\
\% \text { hombres }\end{array}$ & $\begin{array}{l}\text { Media de } \\
\text { edad } \\
\text { (DE o rango), } \\
\text { años }\end{array}$ & $\begin{array}{l}\text { Modelo (tipo) y } \\
\text { duración de la terapia } \\
\text { en el grupo de } \\
\text { intervención }\end{array}$ & $\begin{array}{l}\text { Régimen (sesiones) en el } \\
\text { grupo de intervención y } \\
\text { especialidad del terapista }\end{array}$ \\
\hline $\begin{array}{l}\text { Weinger, } \\
2011^{31}\end{array}$ & $\begin{array}{l}\text { Estados } \\
\text { Unidos }\end{array}$ & $222(50 \%)$ & $52,5 \pm N R$ & $\begin{array}{l}\text { Entrenamiento sobre pautas } \\
\text { de comportamiento estruc- } \\
\text { turadas en } 6 \text { semanas }\end{array}$ & $\begin{array}{l}5 \text { sesiones de } 2 \text { h guiadas por } \\
\text { educadores capacitados }\end{array}$ \\
\hline $\begin{array}{l}\text { Lamers, } \\
2011^{40}\end{array}$ & $\begin{array}{l}\text { Países } \\
\text { Bajos }\end{array}$ & 208 (49\%) & $70,2 \pm 6,60$ & $\begin{array}{l}\text { Acompañamiento de } \\
\text { enfermeras en pacientes } \\
\text { con depresión, de acuerdo } \\
\text { a protocolo MINI durante } \\
6 \text { semanas }\end{array}$ & $\begin{array}{l}4 \text { sesiones de terapia cognitiva } \\
\text { breve de aproximadamente } 1 \\
\text { hora, llevadas a cabo por enfer- } \\
\text { meras certificadas por psiquia- } \\
\text { tras y psicólogos }\end{array}$ \\
\hline $\begin{array}{l}\text { Gregg, } \\
2007^{33}\end{array}$ & $\begin{array}{l}\text { Estados } \\
\text { Unidos }\end{array}$ & $81(53.1 \%)$ & $50,9 \pm N R$ & $\begin{array}{l}\text { Capacitación sumada a } \\
\text { taller ACT (terapia de acep- } \\
\text { tación y compromiso) }\end{array}$ & $\begin{array}{l}\text { Sesiones de } 7 \text { h de educación } \\
\text { sobre morbilidad y } 4 \text { h talleres } \\
\text { ACT, guiados por creador del } \\
\text { modelo o uno de sus discípulos } \\
\text { destacados }\end{array}$ \\
\hline $\begin{array}{l}\text { McGinnis, } \\
2005^{34}\end{array}$ & $\begin{array}{l}\text { Estados } \\
\text { Unidos }\end{array}$ & $39(0 \%)$ & NR & $\begin{array}{l}\text { Protocolo de intervención } \\
\text { en biofeedback y relaja- } \\
\text { ción, ejecutado durante } 3 \\
\text { meses }\end{array}$ & $\begin{array}{l}\text { Entrenamiento semanal en } 10 \\
\text { sesiones de } 45 \text { min, } 5 \text { corres- } \\
\text { ponden a electromiógrafo y } \\
5 \text { biofeedback termal, acom- } \\
\text { pañado de entrenamiento de } \\
\text { relajamiento y } 2 \text { sesiones diarias } \\
\text { autodirigidas de relajación en } \\
\text { casa durante } 15 \text { min }\end{array}$ \\
\hline $\begin{array}{l}\text { Siebolds, } \\
2005^{44}\end{array}$ & $\begin{array}{l}\text { Alema- } \\
\text { nia }\end{array}$ & $223(52 \%)$ & $59,60 \pm 7,10$ & $\begin{array}{l}\text { Programa de acompa- } \\
\text { ñamiento y consejería } \\
\text { durante } 6 \text { meses }\end{array}$ & $\begin{array}{l}\text { Capacitación y entrenamiento } \\
\text { en el uso de implementos para } \\
\text { la autorregulación de los niveles } \\
\text { fisiológicos acompañado de un } \\
\text { sistema de acompañamiento } \\
\text { guiado por el médico, quien fue } \\
\text { capacitado en un sistema de } \\
\text { preguntas específicas adaptadas } \\
\text { para } 4 \text { sesiones en } 24 \text { semanas }\end{array}$ \\
\hline $\begin{array}{l}\text { Gamsu, } \\
2002^{45}\end{array}$ & $\begin{array}{l}\text { Reino } \\
\text { Unido }\end{array}$ & 19 (NR) & $47,88 \pm 8,01$ & $\begin{array}{l}\text { Sesiones grupales de } 2 \mathrm{~h} \\
\text { semanales durante } 12 \\
\text { semanas }\end{array}$ & $\begin{array}{l}\text { Sesiones semanales con énfasis } \\
\text { en autoestima y dieta. Psicólo- } \\
\text { go clínico, enfermera, dietista, } \\
\text { médico }\end{array}$ \\
\hline $\begin{array}{l}\text { Pouwer, } \\
2001^{11}\end{array}$ & $\begin{array}{l}\text { Países } \\
\text { Bajos }\end{array}$ & $300(63.3 \%)$ & $53,50 \pm 17,00$ & $\begin{array}{l}\text { Asesoría complementaria al } \\
\text { tratamiento por } 12 \text { meses }\end{array}$ & $\begin{array}{l}\text { Citas regulares con el internista } \\
\text { cada } 3 \text { o } 4 \text { meses, sumando a } \\
\text { especialistas en casos requeridos. } \\
2 \text { sesiones de } 15 \text { min de trabajo } \\
\text { con enfermeras capacitadas } \\
\text { por psicólogos que monitorean } \\
\text { constantemente los índices de } \\
\text { calidad de vida computarizados }\end{array}$ \\
\hline $\begin{array}{l}\text { Grey, } \\
2000^{35}\end{array}$ & $\begin{array}{l}\text { Estados } \\
\text { Unidos }\end{array}$ & $75(41.4 \%)$ & $14,40 \pm 2,00$ & $\begin{array}{l}\text { Entrenamiento para el } \\
\text { afrontamiento durante } 6 \\
\text { semanas }\end{array}$ & $\begin{array}{l}\text { Sesiones de entre } 60 \text { y } 90 \text { min en } \\
\text { grupos de } 2 \text { o } 3 \text { por entre } 3 \text { y } 5 \\
\text { semanas y seguimiento telefóni- } \\
\text { co una vez al mes por } 12 \text { meses }\end{array}$ \\
\hline $\begin{array}{l}\text { Aikens, } \\
1997^{36}\end{array}$ & $\begin{array}{l}\text { Estados } \\
\text { Unidos }\end{array}$ & $22(41 \%)$ & $61,00 \pm 10,20$ & $\begin{array}{l}\text { Relajación muscular e ima- } \\
\text { ginería por } 8 \text { semanas }\end{array}$ & 6 sesiones, psicólogo clínico \\
\hline
\end{tabular}

NR: No Reportado. PRT: Progressive Resistant Training. ACT: Acceptance and Commitment Therapy. 
Tabla 1. Características de los estudios incluidos en la revisión sistemática (continuación)

\begin{tabular}{|c|c|c|c|c|c|c|c|}
\hline $\begin{array}{l}\text { Año y } \\
\text { referencia }\end{array}$ & $\begin{array}{l}\text { Modelo (tipo) } \\
\text { y duración } \\
\text { de la terapia } \\
\text { en el grupo } \\
\text { control }\end{array}$ & $\begin{array}{c}\text { Régimen } \\
\text { (sesiones) } \\
\text { en el grupo } \\
\text { control y } \\
\text { especialidad } \\
\text { del terapista }\end{array}$ & $\begin{array}{c}\text { Segui- } \\
\text { miento, } \\
\text { meses }\end{array}$ & $\begin{array}{l}\text { Categoría } \\
\text { intervención }\end{array}$ & $\begin{array}{c}\text { Media (DE) \% } \\
\text { de hemoglo- } \\
\text { bina glicada } \\
\text { en la línea } \\
\text { base }\end{array}$ & $\begin{array}{c}\text { Media (DE) \% } \\
\text { de hemoglo- } \\
\text { bina glicada } \\
\text { en segui- } \\
\text { miento }\end{array}$ & $\begin{array}{c}\text { Calidad } \\
\text { metodo- } \\
\text { lógica }\end{array}$ \\
\hline $\begin{array}{l}\text { Weinger, } \\
2011^{31}\end{array}$ & $\begin{array}{l}\text { Psicoedu- } \\
\text { cación por } \\
\text { consulta sin } \\
\text { límite }\end{array}$ & 6 meses & $N R$ & $\begin{array}{l}\text { Psicología } \\
\text { cognitivo- } \\
\text { conductual }\end{array}$ & $9,12 \pm 1,10$ & $8,45 \pm 1,30$ & Alta \\
\hline $\begin{array}{l}\text { Lamers, } \\
2011^{40}\end{array}$ & $\begin{array}{l}\text { Terapia Están- } \\
\text { dar }\end{array}$ & NR & 9 meses & $\begin{array}{l}\text { Psicología } \\
\text { cognitivo- } \\
\text { conductual }\end{array}$ & $7,50 \pm 1,20$ & $7,30 \pm 0,20$ & Alta \\
\hline $\begin{array}{l}\text { Gregg, } \\
2007^{33}\end{array}$ & $\begin{array}{l}\text { Psicoeduca- } \\
\text { ción sin ACT }\end{array}$ & NR & 0 meses & $\begin{array}{l}\text { Psicología } \\
\text { cognitivo- } \\
\text { conductual }\end{array}$ & $8,17 \pm 1,86$ & $7,47 \pm 1,46$ & Alta \\
\hline $\begin{array}{l}\text { McGinnis, } \\
2005^{34}\end{array}$ & $\begin{array}{c}\text { Educación so- } \\
\text { bre diabetes }\end{array}$ & $\begin{array}{c}3 \text { a } 4 \text { sesiones } \\
\text { de } 60 \text { a } 75 \text { min } \\
\text { con } 3 \text { o } 4 \\
\text { semanas de } \\
\text { separación }\end{array}$ & 3 meses & $\begin{array}{l}\text { Psicología } \\
\text { cognitivo- } \\
\text { conductual }\end{array}$ & $7,40 \pm 1,40$ & $6,60 \pm 2,10$ & Baja \\
\hline $\begin{array}{l}\text { Siebolds, } \\
2005^{44}\end{array}$ & $\begin{array}{l}\text { Tratamiento } \\
\text { estándar sin } \\
\text { acompaña- } \\
\text { miento }\end{array}$ & NR & 6 meses & Consejería & $8,47 \pm 0,86$ & $7,47 \pm 1,27$ & Baja \\
\hline $\begin{array}{l}\text { Gamsu, } \\
2002^{45}\end{array}$ & $\begin{array}{l}\text { Evaluación } \\
\text { pre interven- } \\
\text { ción por } 12 \\
\text { semanas }\end{array}$ & NR & 6 meses & $\begin{array}{l}\text { Psicología } \\
\text { cognitivo- } \\
\text { conductual }\end{array}$ & $11,40 \pm 2,60$ & NR & Baja \\
\hline $\begin{array}{l}\text { Pouwer, } \\
2001^{41}\end{array}$ & $\begin{array}{c}\text { Tratamiento } \\
\text { estándar }\end{array}$ & $N R$ & 0 meses & Consejería & $7,80 \pm 1,40$ & $7,70 \pm N R$ & Baja \\
\hline $\begin{array}{l}\text { Grey, } \\
2000^{35}\end{array}$ & NR & NR & $\begin{array}{c}12 \\
\text { meses }\end{array}$ & Consejería & $9,10 \pm 1,50$ & $8,00 \pm 1,20$ & Alta \\
\hline $\begin{array}{l}\text { Aikens, } \\
1997^{36}\end{array}$ & $\begin{array}{l}\text { Terapia están- } \\
\text { dar }\end{array}$ & $N R$ & $\begin{array}{l}8 \text { sema- } \\
\text { nas }\end{array}$ & $\begin{array}{l}\text { Psicología } \\
\text { cognitivo- } \\
\text { conductual }\end{array}$ & $11,00 \pm 1,90$ & $10,20 \pm 1,60$ & Baja \\
\hline
\end{tabular}




\begin{tabular}{|c|c|c|c|c|c|c|c|c|c|c|}
\hline \multirow[b]{2}{*}{ Study or Subgroup } & \multicolumn{3}{|c|}{ Intervention } & \multicolumn{3}{|c|}{ Control } & \multicolumn{2}{|c|}{ Std. Mean Difference } & \multirow{2}{*}{\multicolumn{2}{|c|}{$\begin{array}{l}\text { Std. Mean Difference } \\
\text { IV, Random, } 95 \% \mathrm{Cl}\end{array}$}} \\
\hline & Mean & SD & Total & Mean & SD & Total & Weight & IV, Random, $95 \% \mathrm{Cl}$ & & \\
\hline \multicolumn{11}{|c|}{ 1.2.1 Psicologia cognitivo conductual } \\
\hline Aikens, 1997 & 10.2 & 1.6 & 12 & 11.3 & 1.7 & 10 & $4.6 \%$ & $-0.64[-1.51,0.22]$ & & - \\
\hline Ghezeljeh, 2017 & 7.39 & 1.35 & 30 & 7.35 & 1.35 & 35 & $8.4 \%$ & $0.03[-0.46,0.52]$ & & \\
\hline Gregg, 2007 & 7.47 & 1.46 & 36 & 8.07 & 2.22 & 30 & $8.4 \%$ & $-0.32[-0.81,0.17]$ & & - \\
\hline Lamers, 2011 & 7.3 & 0.2 & 20 & 7.8 & 0.2 & 17 & $4.5 \%$ & $-2.45[-3.32,-1.57]$ & $\longleftarrow$ & \\
\hline Shayeghian, 2016 & 7.03 & 1.52 & 53 & 7.61 & 1.56 & 50 & $9.7 \%$ & $-0.37[-0.76,0.02]$ & & \\
\hline Wayne, 2015 & 7.88 & 1.17 & 48 & 8.13 & 1.27 & 49 & $9.6 \%$ & $-0.20[-0.60,0.20]$ & & - \\
\hline $\begin{array}{l}\text { Weinger, } 2011 \\
\text { Subtotal }(95 \% \mathrm{Cl})\end{array}$ & 8.45 & 1.3 & $\begin{array}{r}66 \\
265\end{array}$ & 8.6 & 1.3 & $\begin{array}{r}70 \\
261\end{array}$ & $\begin{array}{l}10.5 \% \\
55.6 \%\end{array}$ & $\begin{array}{r}-0.11[-0.45,0.22] \\
-0.47[-0.86,-0.07]\end{array}$ & & \\
\hline \multicolumn{11}{|c|}{$\begin{array}{l}\text { Heterogeneity. } \text { Tau }^{2}=0.21 ; \mathrm{Chi}^{2}=26.99, \mathrm{df}=6(\mathrm{P}=0.0001) ; \mathrm{I}^{2}=78 \% \\
\text { Test for overall effect: } Z=2.32(P=0.02)\end{array}$} \\
\hline \multicolumn{11}{|l|}{ 1.2.2 Consejería } \\
\hline Browning, 2016 & 6.67 & 2.09 & 312 & 6.64 & 1.59 & 296 & $12.8 \%$ & $0.02[-0.14,0.18]$ & & - \\
\hline Chen, 2012 & 8.16 & 1.73 & 104 & 8.48 & 1.78 & 110 & $11.5 \%$ & $-0.18[-0.45,0.09]$ & & \\
\hline Grey, 2000 & 7.5 & 1.1 & 41 & 8.5 & 1.4 & 34 & $8.6 \%$ & $-0.80[-1.27,-0.32]$ & & \\
\hline $\begin{array}{l}\text { Siebolds, } 2005 \\
\text { Subtotal }(95 \% \mathrm{Cl})\end{array}$ & 7.47 & 1.27 & $\begin{array}{l}113 \\
570\end{array}$ & 7.81 & 1.52 & $\begin{array}{l}110 \\
550\end{array}$ & $\begin{array}{l}11.5 \% \\
44.4 \%\end{array}$ & $\begin{array}{l}-0.24[-0.51,0.02] \\
-0.24[-0.50,0.03]\end{array}$ & & \\
\hline \multicolumn{11}{|c|}{$\begin{array}{l}\text { Heterogeneity. } \text { Tau }^{2}=0.05 ; \mathrm{Chi}^{2}=11.66, \mathrm{df}=3(\mathrm{P}=0.009) ; \mathrm{I}^{2}=74 \% \\
\text { Test for overall effect: } Z=1.77(P=0.08)\end{array}$} \\
\hline Total $(95 \% \mathrm{Cl})$ & & & 835 & & & 811 & $100.0 \%$ & $-0.35[-0.57,-0.12]$ & & \\
\hline \multicolumn{9}{|c|}{$\begin{array}{l}\text { Heterogeneity: } \text { Tau }^{2}=0.10 ; \mathrm{Chi}^{2}=41.55, \mathrm{df}=10(P<0.00001) ; \mathrm{I}^{2}=76 \% \\
\text { Test for overall effect: } Z=3.00(P=0.003) \\
\text { Test for subqroup differences: } \mathrm{Chi}^{2}=0.91 . \mathrm{df}=1(\mathrm{P}=0.34) . \mathrm{I}^{2}=0 \%\end{array}$} & $\begin{array}{ccc}-1 & -1 & 1 \\
\text { Favours [Intervention] }\end{array}$ & $\begin{array}{cc}1 & 1 \\
0.5 & 1 \\
\text { Favours [Control] }\end{array}$ \\
\hline
\end{tabular}

Figura 4. Análisis según tipo de intervención psicológica.

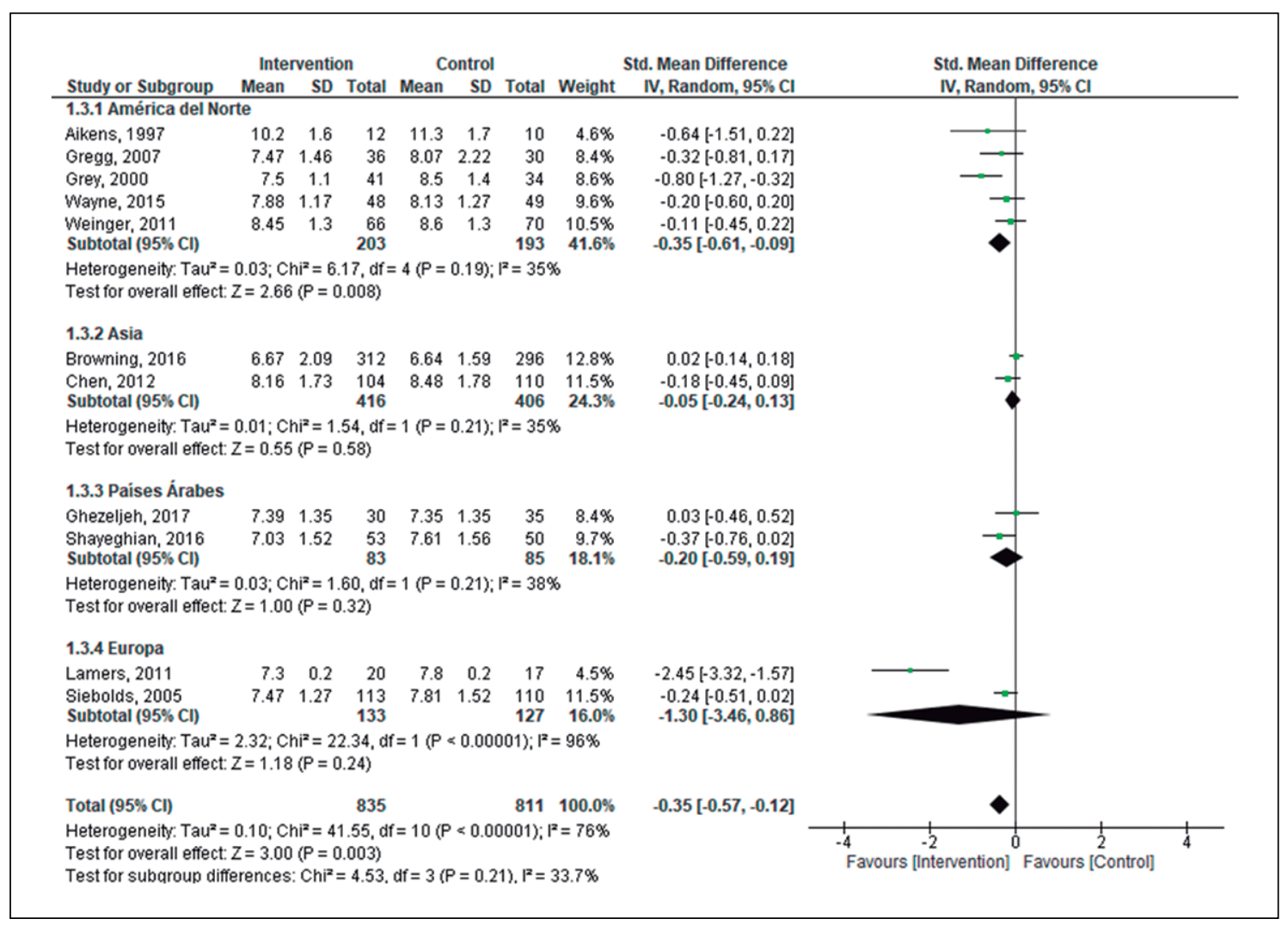

Figura 5. Análisis según origen de los estudios. 
Respecto a la heterogeneidad de los estudios se observó un nivel adecuado en los grupos provenientes de América del Norte, Asia y Países Árabes $\left(I^{2}=35 \% ; I^{2}=35 \% ; I^{2}=38 \%\right.$, respectivamente). Por el contrario, en el grupo de Europa, la heterogeneidad fue considerable $\left(\mathrm{I}^{2}=96 \%, \mathrm{p}<0,001\right)$ (Figura 5).

El análisis de meta-regresión ajustando por duración de la intervención, no arrojó resultados significativos, no obstante, señaló una tendencia $(\mathrm{p}=0,09)$.

\section{Discusión}

Esta revisión da cuenta de una variedad de estrategias utilizadas para enriquecer los tratamientos definidos para la DM2, reflejo de la necesidad de establecer mecanismos más integrales que consideren el proceso de recuperación o afrontamiento de una enfermedad en todas las dimensiones de significado de quien la padezca.

Ante la claridad que existe en torno al impacto que posee en la recuperación el contacto entre el paciente y las instituciones médicas ${ }^{34,35,38-40,44,48}$, llama la atención la falta de inclusión de herramientas tecnológicas que faciliten el seguimiento del estado del paciente por parte del personal médico, o el autocontrol en términos de frecuencia de administración del tratamiento o acceso a información validada sobre la enfermedad, considerando que el uso de estos recursos también podría aumentar la percepción de apoyo recibido en un contexto social cada vez más permeado y familiarizado con las tecnologías de la información y la comunicación ${ }^{34,40,42}$.

Como en otros trabajos ${ }^{14}$, este estudio presenta un número considerable de intervenciones psicológicas implementadas en pacientes diabéticos desde la perspectiva cognitivo conductual ${ }^{32-34,36-38,40,45,48}$, aunque también destacan las intervenciones de consejería para el autocuidado, la generación de disciplinas y la mejora de la calidad de vida desde las relaciones sociales ${ }^{35,39,41,44,47}$, junto con, aquellas referidas al uso de técnicas de meditación, que pueden ser entendidas desde la psicología transpersonal ${ }^{31,46}$.

No obstante, los resultados de los estudios analizados dejan claro que la implementación de estrategias basadas exclusivamente en las condiciones psicológicas o sociales del indivi- duo $^{31,41,42,46}$ no son suficientes para producir una mejora significativa en los indicadores biológicos que logre justificar los esfuerzos terapéuticos de la intervención, aun cuando el análisis de meta-regresión sugiere que la mayor duración de la misma reforzaría su impacto. En este sentido, el éxito de este enfoque complementario al tratamiento, recae en la transformación de las creencias y responsabilidades asignadas por cada paciente a los mecanismos de regulación de los niveles de glicemia, que incluyen tanto el autocontrol de la ingesta alimentaria, actividad física y el cuidado de las relaciones sociales, llegando incluso al trabajo espiritual como herramienta significativa en algunos pacientes, elementos que no se restringen a la mera educación sobre la condición, características y necesidades asociadas, sino que, tienen más que ver con el desarrollo de una conciencia de enfermedad clara, sumado a patrones de acción concretos, que le permitan al individuo hacerse cargo de su propio proceso de mejora, con la seguridad de contar con un espacio estructurado y constante de apoyo ${ }^{34,40,43,48}$.

Finalmente, cabe señalar que estas intervenciones psicológicas podrían ser aplicadas en el box de atención por psicólogos incorporados en equipos interdisciplinarios ${ }^{49}$, pero también por distintos profesionales debidamente entrenados dependiendo de la complejidad del enfoque terapéutico y del nivel de entrenamiento requerido ${ }^{50}$.

\section{Conclusión}

La evidencia analizada en este estudio, sugiere que el apoyo con herramientas psicológicas al tratamiento médico regular de los pacientes con DM2, podría ser un complemento efectivo para reducir los niveles de hemoglobina glicada, potenciando elementos como la autorregulación, la conciencia de la enfermedad y la adherencia desde la perspectiva de la percepción de autoeficacia.

Como limitación de este trabajo se debe señalar la cantidad de estudios con datos disponibles para realizar una integración metanalítica. Esto, podría significar la pérdida de alguna experiencia relevante que no haya sido incorporada por falta de información. Además, se debe considerar que los tipos de intervenciones, dada la temática, son muy disímiles lo cual dificultó su sistematización. 


\section{Referencias}

1. Federación Internacional de la Diabetes. Atlas de la Diabetes de la FID. [Internet]. Séptima edición. International Diabetes Federation; 2015 [Citado el 10 septiembre de 2019]. Disponible en: https://www.fundaciondiabetes.org/upload/publicaciones_ficheros/95/ IDF_Atlas_2015_SP_WEB_oct2016.pdf.

2. Federación Internacional de la Diabetes. Atlas de la Diabetes de la FID. [Internet]. Octava edición. International Diabetes Federation; 2017. [Citado el 10 septiembre de 2019]. Disponible en: http://diabetesatlas.org/IDF_Diabetes_Atlas_8e_interactive_ES/.

3. Ministerio de Salud, Gobierno de Chile. Encuesta Nacional de Salud ENS Chile 2009-2010. Santiago: Ministerio de Salud; 2011.

4. Comunidad de Madrid. Estrategia de atención a pacientes con enfermedades crónicas en la Comunidad de Madrid. Madrid: Consejería de Sanidad; 2013.

5. Organización Panamericana de la Salud. Cuidados innovadores para las condiciones crónicas: Organización y prestación de atención de alta calidad a las enfermedades crónicas no transmisibles en las Américas. Washington: Organización Panamericana de la Salud; 2013.

6. Wagner EH, Austin BT, Von Korff M. Organizing care for patients with chronic illness. Milbank Q 1996: 51144.

7. Wagner EH, Austin BT, Davis C, Hindmarsh M, Schaefer J, Bonomi A. Improving chronic illness care: translating evidence into action. Health Aff (Millwood) 2001; 20(6): 64-78.

8. Wagner EH. Chronic disease management: what will it take to improve care for chronic illness? Eff Clin Pract 1998; 1 (1): 2-4.

9. Singh D, Ham C. Improving care for people with long term conditions: a review of UK and international frameworks. 2006. Birmingham: NHS Institute for Innovation and Improvement Google Scholar; 2008.

10. Piatt GA, Orchard TJ, Emerson S, Simmons D, Songer TJ, Brooks MM, et al. Translating the chronic care model into the community: results from a randomized controlled trial of a multifaceted diabetes care intervention. Diabetes Care 2006; 29 (4): 811-7.

11. Bodenheimer $\mathrm{T}$, Wagner $\mathrm{EH}$, Grumbach K. Improving primary care for patients with chronic illness. JAMA 2002; 288 (14): 1775-9.

12. Uchendu C, Blake H. Effectiveness of cognitive-behavioural therapy on glycaemic control and psychological outcomes in adults with diabetes mellitus: a systematic review and meta-analysis of randomized controlled trials. Diabet Med 2017; 34 (3): 328-39.
13. Ismail K, Winkley K, Rabe-Hesketh S. Systematic review and meta-analysis of randomised controlled trials of psychological interventions to improve glycaemic control in patients with type 2 diabetes. Lancet 2004; 363 (9421): 1589-97.

14. Alam R, Sturt J, Lall R, Winkley K. An updated meta-analysis to assess the effectiveness of psychological interventions delivered by psychological specialists and generalist clinicians on glycaemic control and on psychological status. Patient Educ Couns 2009; 75 (1): 25-36.

15. Liberati A, Altman DG, Tetzlaff J, Mulrow C, Gotzsche PC, Ioannidis JP, et al. The PRISMA statement for reporting systematic reviews and meta-analyses of studies that evaluate health care interventions: explanation and elaboration. PLoS Med 2009; 6 (7): e1000100.

16. Strawbridge S, Woolfe R. Counselling psychology: Origins, developments and challenges. Tercera edición. London: SAGE; 2010.

17. Ruiz M, Díaz M, Villalobos A. Manual de técnicas de intervención cognitivo conductuales. Bilbao: Desclée de Brouwer; 2012.

18. Grof S. Breve historia de la psicología transpersonal. J Transpersonal Res 2010; 2 (2): 125-36.

19. Rafalin D. Counselling psychology and research: Revisiting the relationship in the light of our 'mission'. Therapy and beyond: Counselling psychology contributions to therapeutic and social issues. Oxford: Wiley-Blackwell; 2010.

20. Wilson KG, Soriano MC. Terapia de aceptación y compromiso (ACT): Madrid: Ediciones Pirámide; 2002.

21. Coutiño AM. Terapias cognitivo-conductuales de tercera generación (TTG): la atención plena/mindfulness. Rev Intern Psicología 2012; 12 (01).

22. Barraca J. ¿Aceptación o control mental? Terapias de aceptación y mindfulness frente a las técnicas cognitivo-conductuales para la eliminación de pensamientos instrusos. Análisis y Modificación de Conducta 2011; 37 (155): 43-63.

23. Grof C, Grof S. Spiritual emergency: The understanding and treatment of transpersonal crises. International Journal of Transpersonal Studies 2017; 36 (2): 5.

24. Canda ER, Smith ED. Transpersonal perspectives on spirituality in social work. New York: Routledge; 2013.

25. Almendro M. Psicología y psicoterapia transpersonal. España: Editorial Kairós; 2012.

26. Jadad AR, Moore RA, Carroll D, Jenkinson C, Reynolds DJ, Gavaghan DJ, et al. Assessing the quality of reports of randomized clinical trials: is blinding necessary? Control Clin Trials 1996; 17 (1): 1-12.

27. Cerda J, Villarroel L. Evaluación de la concordancia 
inter-observador en investigación pediátrica: Coeficiente de Kappa. Rev Chil Pediatr 2008; 79 (1): 54-8.

28. Niemeyer H, Musch J, Pietrowsky R. El sesgo de publicación en metaanálisis sobre la eficacia de las intervenciones psicoterapéuticas para la Esquizofrenia. RET Revista de Toxicomanías 2014; 73: 32-48.

29. Ryan R. Heterogeneity and subgroup analyses in cochrane consumers and communication review group reviews: planning the analysis at protocol stage. [Internet]. Cochrane Consumers and Communication Review Group; 2013. [Citado el 10 de septiembre de 2019]. Disponible en: https://cccrg.cochrane.org/sites/cccrg. cochrane.org/files/public/uploads/heterogeneity_subgroup_analyses_revising_december_1st_2016.pdf.

30. Centro Cochrane Iberoamericano. Manual Cochrane de Revisiones Sistemáticas de Intervenciones, versión 5.1. 0. [Internet]. Barcelona: Centro Cochrane Iberoamericano; 2012. [Citado el 10 septiembre de 2019]. Disponible en: https://es.cochrane.org/sites/es.cochrane.org/files/ public/uploads/manual_cochrane_510_web.pdf.

31. Weinger K, Beverly EA, Lee Y, Sitnokov L, Ganda OP, Caballero AE. The effect of a structured behavioral intervention on poorly controlled diabetes: A randomized controlled trial. Arch Intern Med 2011; 171 (22): 19909.

32. Putiri AL, Lovejoy JC, Gillham S, Sasagawa M, Bradley R, Sun G-C. Psychological Effects of Yi Ren Medical Qigong and Progressive Resistance Training in Adults With Type 2 Diabetes Mellitus: A Randomized Controlled Pilot Study. Altern Ther Health Med 2012; 18 (1): 30-4.

33. Gregg JA, Callaghan GM, Hayes SC, Glenn-Lawson JL. Improving diabetes self-management through acceptance, mindfulness, and values: a randomized controlled trial. J Consult Clin Psychol 2007; 75 (2): 336-43.

34. McGinnis RA, McGrady A, Cox SA, Grower-Dowling KA. Biofeedback-assisted relaxation in type 2 diabetes. Diabetes Care 2005; 28 (9): 2145-9.

35. Grey M, Boland EA, Davidson M, Li J, Tamborlane WV. Coping skills training for youth with diabetes mellitus has long-lasting effects on metabolic control and quality of life. J Pediatr 2000; 137 (1): 107-13.

36. Aikens JE, Kiolbasa TA, Sobel R. Psychological predictors of glycemic change with relaxation training in non-insulin-dependent diabetes mellitus. Psychother Psychosom 1997; 66(6): 302-6.

37. Shayeghian Z, Hassanabadi H, Aguilar-Vafaie ME, Amiri P, Besharat MA. A Randomized Controlled Trial of Acceptance and Commitment Therapy for Type 2 Diabetes Management: The Moderating Role of Coping Styles. PloS One 2016; 11 (12): e0166599-e.
38. Ghezeljeh TN, Kohandany M, Oskouei FH, Malek M. The effect of progressive muscle relaxation on glycated hemoglobin and health-related quality of life in patients with type 2 diabetes mellitus. Appl Nurs Res 2017; 33: 142-8.

39. Ebrahimi H, Sadeghi M, Amanpour F, Vahedi H. Evaluation of empowerment model on indicators of metabolic control in patients with type 2 diabetes, a randomized clinical trial study. Prim Care Diabetes 2016; 10 (2): 129-35.

40. Lamers F, Jonkers CC, Bosma H, Knottnerus JA, Van Eijk JT. Treating depression in diabetes patients: does a nurse-administered minimal psychological intervention affect diabetes-specific quality of life and glycaemic control? A randomized controlled trial. J Adv Nurs 2011; 67 (4): 788-99.

41. Pouwer F, Snoek FJ, Van der Ploeg HM, Ader HJ, Heine RJ. Monitoring of psychological well-being in outpatients with diabetes: effects on mood, $\operatorname{HbA}(1 \mathrm{c})$, and the patient's evaluation of the quality of diabetes care: a randomized controlled trial. Diabetes Care 2001; 24 (11): 1929-35.

42. Browning C, Chapman A, Yang H, Liu S, Zhang T, Enticott JC, Thomas SA. Management of type 2 diabetes in China: the Happy Life Club, a pragmatic cluster randomised controlled trial Browning, Colette using health coaches. BMJ Open 2016; 6 (3): e009319-e.

43. Wayne N, Pérez DF, Kaplan DM, Ritvo P. Health Coaching Reduces HbAlc in Type 2 Diabetic Patients From a Lower-Socioeconomic Status Community: A Randomized Controlled Trial. J Med Internet Res 2015; 17 (10): e224-e.

44. Siebolds M, Gaedeke O, Schwedes U. Self-monitoring of blood glucose-psychological aspects relevant to changes in HbAlc in type 2 diabetic patients treated with diet or diet plus oral antidiabetic medication. Patient Educ Couns 2006; 62 (1): 104-10.

45. Gamsu DS, Sutton MS, Bennett L, Ward JD. The development of a psychoeducational group intervention for overweight women with type 2 diabetes mellitus: A service evaluation. Practical Diabetes International 2002; 19 (2): 43-50.

46. Fall E, Roche B, Izaute $M$, Batisse $M$, Tauveron I, Chakroun N. A brief psychological intervention to improve adherence in type 2 diabetes. Diabetes Metab 2013; 39 (5): 432-8.

47. Chen SM, Creedy D, Lin HS, Wollin J. Effects of motivational interviewing intervention on self-management, psychological and glycemic outcomes in type 2 diabetes: A randomized controlled trial. Int J Nurs Stud 2012; 49 (6): 637-44.

48. Koloverou E, Tentolouris N, Bakoula C, Darviri C, 
Chrousos G. Implementation of a stress management program in outpatients with type 2 diabetes mellitus: a randomized controlled trial. Hormones (Athens) 2014; 13 (4): 509-18.

49. Grau J. Enfermedades crónicas no transmisibles: un abordaje desde los factores psicosociales. Salud \& Sociedad 2016; 7 (2): 138-66.

50. González-Cantero J, Oropeza R. Intervenciones cognitivo conductuales para diabéticos en México. Salud Mental 2016; 39 (2): 99-105. 